\title{
Study on Stock Selection Strategy Based on SPSS Regression Mean Quantization
}

\author{
Wenzhong Fan ${ }^{1}$ \\ ${ }^{1}$ School of management, Donghua University, 200051 Shanghai City China \\ Correspondence: Wenzhong Fan, School of management, Donghua University, 200051 Shanghai City China. E-mail: \\ wenzhongfan@163.com
}

Received: August 6, 2016

Accepted: August 17, 2016

Online Published: September 13, 2016

doi:10.5430/bmr.v5n3p26

URL: http://dx.doi.org/10.5430/bmr.v5n3p26

\begin{abstract}
In the stock market, the main three kinds of state, rise, decline and the range of volatility, in the technical analysis indicators are MACD, KDJ, and other combinations. In the specific investment, technical analysis often has limitations such as delay or delay. In this paper, through the analysis of the various indicators of the combination, a new focus on the filter method, selection of different industries, a comprehensive comparison of the effectiveness of indicators. Based on the 30 day moving average, the extreme values of different technical indicators are selected, and the corresponding effective indexes are selected for clustering analysis.
\end{abstract}

Keywords: Component, SPSS, Stock, Principal component analysis, Cluster analysis

\section{Introduction}

Stock is the product of the market economy, investors are trying to predict the development trend of stock. However, the stock market as a giant system under the influence of many uncertain factors, the volatility of the stock price tends to show strong nonlinear characteristics. At present, there are many methods for the prediction of the stock price, at the end of the seventies of the 20th century "Elliott Wave Analysis and the Gann method including Robert D. Edwards and John McGee's trend of stock technical analysis and Richard Vakov etc. are used to be all the rage.

Based on the CARR model, this paper uses the model to fit the stock price fluctuation, and decomposes the trading volume of the volatility period, and introduces the model of the trading volume. Finally, the accuracy of the introduction of the daily fluctuation data is introduced to improve the accuracy of the volume and price. Stock price analysis is based on the process to study the price change law. Trading volume in the stock market plays an important role in the stock market as a result of the CARR model, [1] which is more robust than the GARCH model in volume and price volatility. So this paper attempts to import different volume factor in CARR model, for the stock market has long memory. Long memory refers to the distance between the time interval is still significant, that is, the impact of the past will continue to affect the future. By using different long memory test methods for the Shanghai and Shenzhen stock markets, the yield sequence is tested, and the time interval of time series is different from that of the time period. Therefore, based on the transaction volume, it is converted to the trading volume as a measure of the basis, with the linear function and the function of the process of the transaction volume and time course of the conversion, with the MDH theory information, and is also the basis of the dynamic process of the transaction. Based on the variance of the method, the continuous trading volume in different time periods of the stock price and the volatility of the index is explained. Selecting an appropriate model for predicting the investors' behavior is a challenging task and may require a variety of tests in order to make a decision. Comparing competing models can be done by using performance measures or by implementing other innovative comparison tools (Riasi and Wang, 2016)"

\section{The Characteristics and Development of CARR}

Model Multivariate CARR model, Chou (2015) proposed the CARR model, Here, the T is the conditional mean of all information before the time $t$, the distribution of the interference terms is assumed to be the distribution of the density function of the mean value of a unit. Coefficients ((I, J, beta) are all positive for the positive. If it is I, I, D, [4] the conditional variance is proportional to the mean square of its condition. If it is not, then the distribution of the mean and variance of the mean and the variance of the mean value is non-negative. The conditional variance of the Carr model can be extended to multiple factors, and $\mathrm{Xt}(1=1,2,3 \ldots \mathrm{L})$, then the condition equation is changed into the following form:

Then the model is a multivariate CARR model, that is, the CARRRX model, if the perturbation terms are exponentially 
distributed, the model is called the E-CARRX model. Some important exogenous variables, such as volume, continuous time, and periodic factors, etc.. The average number of the volume and the total volume of the total volume of the total number of errors correction whether it is the error correction term before the coefficient or the pre exponential price of the previous factor.

Two empirical analysis

In this paper, we choose the composite index and stock, the sample interval is 2005-2015, the daily closing price, the highest price, the lowest and the volume as the statistics, the daily trading volume of the unit is the sum of the number, data sources and wind consulting, the use of E views 5 software to analyze the model.

The formula for calculating the range and yield of the paper is as follows: $y t=100^{*}$ (phight-ptlow) Yield $\mathrm{RT}=100^{*} \ln$ (pt/pt-1), here, Pt is the daily closing price of each trading day, the highest price per day is the value of the lowest stock price of each trading day.

Empirical test on the dynamic relationship between volatility of stock volume and stock index

In the CARR- $(x, y)$ model, the conditional variance equation of different types of trading volume, the model one is: $\sigma 2 t=h t+\theta 1 V t$

From the empirical results of the above models, the following conclusions can be drawn: the rise of the volatility of the cycle is 0.95 and the average is 0.34 , which indicates that the average trading volume and the time series are very strong and positive correlation.

1) after two types of trading volume, the value of $\mathrm{X}$ and $\mathrm{y}$ are basically smaller, which shows that trading volume has a certain ability to explain the volatility of the stock price, and the volume of the stock has been reduced to a certain extent.

2) compared with the time coefficient, theta 1 than 2 to and theta 2 in t statistic on very significant. The above shows that the long-term volume on the volatility of stock price has some explanatory power, short-term trading volume on the stock price volatility of did not explain.

3) the results of the estimation of the model, and the number of the long-term trading volume exceeds the value of the system of the previous model, the numerical value of $\mathrm{T}$ is very significant, and the value of the alpha + beta is also reduced to 0.4799 . In the long term, the long-term trading volume is more than the mean value of the stock price volatility has a strong explanation.

4) after the addition of the volume, the volatility of the stock market has not completely disappeared, which shows that the stock market is not fully reflected in the volume of information, there are other factors that affect the volatility of the stock market. This is due to the impact of many factors, including macroeconomic situation, industry conditions, the company fundamentals, market microstructure, the introduction of relevant policies, investors' psychological expectations.

a) VCARR model is more robust than the traditional MARR model in the dynamic relationship between volume and stock price volatility.

b) the empirical analysis is carried out on the conditional variance equation of the different types of trading volume as the exogenous variable to the CARR model. The results show that: the first short-term trading volume has no explanatory effect on the volatility of stock price; the second is that the long-term trading volume has a certain effect on the volatility of stock price, while the short-term trading volume is not explained.

Draw the following conclusions

c) volume in different time series of yield related. The objective existence of the phenomenon of asynchronous transaction makes the improvement of the statistical properties of stock returns from the stock market. Speed and duration of measurement of time series information.

d) trading volume is related to the volatility of different time periods. When the time series of 3-9 cycle, the continuous combination of time series, the selling non continuous combination can be obtained by the $2 \%$ of the week. According to the model, the results show that the maximum value of the intermediate momentum, and the extreme value of the amount of the varieties, can obtain excess returns. From the volatility of high and low level to choose the good rate to treat the market as a proxy variable GARCH model to introduce the volatility equation to make the volatility of earnings volatility, which means that the market flow of information into the sequence of stock returns, resulting in the ARCH effect. Volume contains important information about the future price movements.

the dynamic relationship between continuous volume and stock index volatility Moudle $2 \sigma 2 t=h t+\theta 4 V u t+V 5 e t$ 
The CARR model is used to study the relationship between quantity and price, and it is found that both the volume and the index of the stock are well explained. The applicability of the mixed distribution hypothesis theory based on the theory of volume and price relationship in CARR model. In view of the GARCH effect of stock price fluctuations, it is found that the current trading volume has a significant effect on the GARCH effect, that is, the volatility of the financial market and the frequency of the impact of information market is highly relevant. A study on the 20 stocks and the stock index of the Chinese stock market. It is also obvious that the GARCH effect of the transaction volume has obvious effect on the difference between the stock index and the stock market. This is because the stock index actually often has an offsetting effect on the stock price, and the market transaction volume is always linear. Using the stochastic volatility (SV) model and Bayesian estimation method based on Markov Monte Carlo simulation (MCMC), the transaction volume is decomposed into expected and unexpected trading volume. This is also verified by the mixed distribution hypothesis (MDH) theory. According to the mixed distribution hypothesis, the trading volume can explain the price volatility. But not fully absorb the price fluctuations of the continuity. Different markets, the volume of transactions on the price volatility of the interpretation of different. The trading volume series and price series are the sample, and the trading volume is more powerful to explain the price fluctuation. Long term time series to control the short - time series, the need to determine the long-term trading and short-term trading agreement. And the effectiveness of the Chinese stock market is the worst. This may be closely related to the factors such as policy market and excessive speculation in the Chinese stock market, which is the time trend, self correlation and volatility. The mixed distribution hypothesis (MDH) is proposed for the first time. The income and the trade volume of the financial assets is determined by a potential non observable information flow variable. The impact of the information flow will produce the change of the income and the transaction volume. In the condition variance equation of the GARCH model, the coefficient of the transaction volume is very significant, and the impact factor of the past is no longer significant. The trading volume is driven by the same factors that produce price fluctuations. Some of the stock market in China has a significant leverage effect, and the non expected trading behavior has a non - symmetrical characteristic of the impact of market volatility. Positive trading volume impact (trading volume impact) impact on market volatility than the same degree of negative trading volume (volume shrinkage shock) shock. [5]The improvement of the statistical properties of the stock price sequence of the trading volume has the objective connection between the dynamic equation and the volume of the stock price.

\section{the effect of continuous volume and the dynamic relationship between volume and stock Moudle $3 \sigma 2 t=h t+\theta 6$ VutIt}

The card test, variance analysis and cross spectrum analysis and a series of methods to study the relationship between volume and price, first of several relationships between volume and price of summary: prices rise; volume down; volume rise. price down There is a positive relationship between the yield and the amount of the transaction, and the positive relationship between the yield and the transaction amount is positive. There is a significant correlation between the real quantity and price, and through the Dynamic Empirical Analysis of the relative contribution rate curve of the trade volume, it can be seen that the introduction of the random variable is appropriate. A time unit as a time period as the volume integration and change to do as a result of variable with price fluctuations in the use of indicators, to determine the long-term popularity of the gathering, from the perspective of the amount of the stock market in china. In the bull market, the forecast of the future with the volume of the past has a certain predictability.

At the same time when stocks into. Volatility persistence (persistence) or volatility accumulation (clustering). [3]The relationship between stock value information and price fluctuation is studied, and the daily trading volume is introduced. It can be found that volatility and trading volume for an effective. Stock indexes are often offset by the larger price fluctuations, but the total market volume is not such an offsetting effect, is always linear positive superimposed, so that the relationship between the volatility of trading volume and yield volatility, even if there is a significant relationship between the amount of trading volume and volatility, but for the entire market index and total transaction volume is not necessarily so.

Volume support and drive prices. Volume is not the Shanghai Composite Index closing price of the Granger cause, the index does not have an explanation of the volume. Only in the case of the bull market, cause trading volume is the Shanghai Composite Index closing price of Granger .

Both the financial and economic composite index and the volume series are stationary series, and the yield and the sequence of the trading volume are bidirectional Granger causality, and the transaction volume information contains the direction and the magnitude of the change in the future. In predicting future changes, stock prices and trading volume are very important. Using Volume EGARCH- $(\mathrm{GARCH})$ model, in the stock market development of the transition, adjustment phase (1993-1996), the same period as the information arrival of the proxy variable, significantly reduce the volatility of the Shanghai and Shenzhen two, and in the specification, the development stage (19972001) and Shanghai and Shenzhen two trading volume cannot explain the volatility of the volatility of the sustainability of the impact of other 
factors. The mixed distribution hypothesis shows that the explanatory power of the stock market is different, and the mechanism of the impact of the information on the volatility of the stock market is changing with the development of the stock market. In the framework of the market microstructure, the ARCH effect is proposed, and the speed of information arrival is determined by the information arrival rate and the information exchange rate in the market. [2]Volume can explain a considerable number of conditional return variance, partial shares can be fully explained. The Carr model research the effect of market in the early and middle volume of price volatility fell, found in the conditional variance equation including spot trading volume can significantly reduce the stock return volatility persistence. Using linear model, the relationship between the amount and price of the 1 stocks were tested. It is found that the absolute quantity of daily trading volume and price changes are positively related to the price changes. The results show that the trading volume of the Chinese stock market can explain the persistence of the volatility of returns. At the same time, as a new market, China's stock market presents the characteristics of the yield and volatility, the stock market can not represent the overall sample. Test results are not robust. The stock market has the characteristics of high growth and volatility, the stock market return and volatility may be different characteristics, so it is necessary to divide the stages of the stock market development, so as to further investigate the impact of trading volume on the volatility of the stock market volatility. Found that the volatility of the CARR model in the introduction of the trading volume of the majority of the volatility of the stock continued to disappear. This also shows that the mixed distribution hypothesis (MDH) is not used to explain the ARCH effect of all securities market volatility, which is a limitation of the mixed distribution hypothesis.

comparison of different models

Through the above information and empirical analysis, the path of the research is based on the volatility and the rate of return and change at different times. These studies show that trading volume is one of the causes of stock price fluctuation, and it has a certain memory cycle, and the impact of a quantity on the price fluctuations in the memory cycle will be reflected.

\section{Conclusion}

Through the relationship of China's stock market overall price volume relationship in the consolidation of market, the bull market and bear market three kinds of different background two analysis, mainly get the following results:

1) there is a cointegration relationship between the price and the volume in any stage of the market, which indicates that there is a balance between the amount and price, and it is positively related to the index and the turnover in the long term.

2) at any stage of the market, the price is not only cause.

In the future, The research on the establishment model of more influence factors will be the research direction of this paper.

\section{References}

Ding Zhongming, Xia Wanjun. (2005). Analysis of CARR model of China's stock market volatility. Business Economics and management, (12), 41-45.

Ray Y, Forecasting, Chou. (2005). financial Volatilities with ext evaluate: the conditional autoregressive range(CARR)mo del. Values: the conditional autoregressive range mo (CARR) del. rem. Journal of Money, Credit and Banking, (37), 561 -582.

Riasi, A., \& Wang, D. (2016). Comparing the Performance of Different Data Mining Techniques in Evaluating Loan Applications. International Business Research, 9(7), 164-187. http://dx.doi.org/10.5539/ibr.v9n7p164

summer days. (2007). The dynamic relationship between volume and stock price volatility based on CARR model. Quantitative statistics and management, (5), 22 -33.

Wang Jintian, Zhao Songshan. (2004). The relationship between the stock market and the volatility of the stock market and the trading volume. Journal of Liaoning Technical University: Social Science Edition.

Xiatian. (2007). The dynamic relationship between volume and stock price volatility based on CARR model. The number of statistics and management, (5), 22 -33. 